\section{Janibacter species with evidence of genomic polymorphism isolated from resected heart valve in a patient with aortic stenosis}

\author{
Lile Malania, ${ }^{1}$ Ying Bai, ${ }^{2}$ \\ Kamil Khanipov, ${ }^{3}$ Marika Tsereteli, ${ }^{4}$ \\ Mikheil Metreveli, ${ }^{5}$ David Tsereteli, ${ }^{1}$ \\ Ketevan Sidamonidze, ${ }^{1}$ Paata Imnadze, ${ }^{1}$ \\ Yuriy Fofanov, ${ }^{3}$ Michael Kosoy ${ }^{2}$ \\ ${ }^{1}$ National Center for Disease Control \\ and Public Health, Tbilisi, Georgia; \\ ${ }^{2}$ Division of Vector-Borne Diseases, \\ Centers for Disease Control and \\ Prevention, Fort Collins, CO, USA; \\ ${ }^{3}$ Department of Pharmacology and \\ Toxicology, University of Texas Medical \\ Branch, Galveston, TX, USA; ${ }^{4}$ Tbilisi \\ State Medical University, Tbilisi, \\ Georgia; ${ }^{5}$ Department of Cardiology, \\ High Technology Medical Center, \\ University Clinic, Tbilisi, Georgia
}

\begin{abstract}
The authors report isolation and identification of two strains of bacteria belonging to the genus Janibacter from a human patient with aortic stenosis from a rural area of the country of Georgia. The microorganisms were isolated from aortic heart valve. Two isolates with slightly distinct colony morphologies were harvested after sub-culturing from an original agar plate. Preliminary identification of the isolates is based on amplification and sequencing of a fragment of 16SrRNA. Whole genome sequencing was performed using the Illumina MiSeq instrument. Both isolates were identified as undistinguished strains of the genus Janibacter. Characterization of whole genome sequences of each culture has revealed a $15 \%$ difference in gene profile between the cultures and confirmed that both strains belong to the genus Janibacter with the closest match to J. terrae. Genomic comparison of cultures of Janibacter obtained from human cases and from environmental sources presents a promising direction for evaluating a role of these bacteria as human pathogens.
\end{abstract}

\section{Introduction}

Bacteria are a common source of infective endocarditis with inflammation of the endothelia cells and valves of the heart, usually caused by bacteria. ${ }^{1}$ Blood culture is the most reliable diagnostic tool. However, sometimes a diagnosis cannot be made using blood culture because of poor laboratory infrastructure, presence of uncommon pathogens, or pretreatment of the patient with antibiotics prior to sample collection. Up to $35 \%$ of all infective endocarditis cases remain blood culture negative either due to slow growth of the bacteria or inappropriate media. $^{2}$

Calcific aortic stenosis is also a major problem in many countries. In the United States, the incidence rate of aortic stenosis has been reported as 27.1 per $10,000 .^{3}$ The mechanism of calcification remains unclear. The hypothesis is that low grade chronic or recurrent bacterial endocarditis with specific calcifiable bacteria is a cause of calcification of the aortic valves. ${ }^{4}$ Along those lines, an association between Chlamydia pneumonia and Mycoplasma pneumonia and calcification in aortic stenosis has been reported.5,6 More recently, a case of Bartonella endocarditis on a bioprosthetic aortic valve that caused rapidly progressive aortic stenosis was reported.?

Due to recent descriptions of Bartonella infections in the country of Georgia ${ }^{8}$ and the importance of identifying sources of bacterial endocarditis, a collaboration was established between hospitals in Tbilisi (the capital of Georgia) and the National Center for Disease Control and Public Health (NCDC\&PH), where culturing of fastidious microorganisms from endocarditis cases has been performed. In this paper, we report isolation and identification of two bacterial strains, both belonging to the genus Janibacter, from heart valve tissues collected during an investigation of a human case, which originally was suspected as endocarditis, but later diagnosed as a case of aortic stenosis. Detailed characterization of the full genomes of the both isolates was performed with identification of a significant difference in gene composition between cultures of these two Janibacter.

\section{Materials and Methods}

\section{Bacterial culture}

Surgically removed cardiac valve material and whole blood were collected from a patient with suspected endocarditis. The clinical part of the work was approved by the Ethical Committee of the High Technology Medical Center, Tbilisi, Georgia. The material was frozen, transferred to the National Center for Diseases Control and Public Health, Tbilisi, Georgia, and stored at $-80^{\circ} \mathrm{C}$ until it was plated. The homogenized cardiac tissue and whole
Correspondence: Ying Bai, Division of Vector-Borne Diseases, Centers for Disease Control and Prevention, 3156 Rampart Road, Fort Collins, Colorado 80521 USA.

Tel. 1-970-266-3555.

E-mail: bby5@cdc.gov

Key words: aortic stenosis; bacteria; genome polymorphism; Janibacter.

Acknowledgements: The authors would like to thank Natalia Abazashvili, Tamriko Giorgadze, and Nazibrola Chitadze for their assistance with laboratory tests; Guram Katstadze, and Neli Chakvetadze for their participation in investigation of the human case; Levent Albayrak and George Golovko for the assistance with genome analysis; and Christina Nelson for careful revision of the manuscript, thoughtful comments, and constructive suggestions.

Contributions: LM, YB, and KK contributed equally. LM, MK, conception and design; LM, MT, MM, DT, data collecting; LM, KS, bacteriological analysis; YB, KS, sequence analyses; KH, YF, genome analysis; LM, YB, MK, manuscript writing; $\mathrm{MK}$, PI, manuscript reviewing and final approval

Conflict of interests: the authors declare no potential conflict of interests.

Funding: the work was supported by the International Science and Technology Center Project (ISTC G-1683-p).

Received for publication: 1 April 2019. Revision received: 10 July 2019

Accepted for publication: 11 July 2019.

This work is licensed under a Creative Commons Attribution-NonCommercial 4.0 International License (CC BY-NC 4.0).

${ }^{(} C$ Copyright: the Author(s), 2019

Licensee PAGEPress, Italy

Infectious Disease Reports 2019; 11:8132

doi:10.4081/idr.2019.8132

blood were used to inoculate chocolate agar plates. The inoculated plates were incubated at $35^{\circ} \mathrm{C}$ with $5 \%$ carbon dioxide for 30 days. Each individual colony from agar was separately streaked onto secondary agar plates. The subcultures were harvested separately by scraping bacterial growth after sufficient growth was observed. DNA was heat extracted at $95^{\circ} \mathrm{C}$ for $10 \mathrm{~min}$ from whole bacterial cells. The prepared DNA was stored at $-80^{\circ} \mathrm{C}$.

\section{PCR amplification and sequencing}

Amplification of the 16S rRNA fragments from isolated DNA of both cultures 
was performed by conventional broadrange PCR assay using $63 \mathrm{~F}$ and $1187 \mathrm{R}$ primers in a $\mathrm{C} 1000$ Touch Thermal Cycler (Bio-Rad, Hercules, CA). The PCR products were analyzed for the presence of amplicons of the appropriate size by electrophoresis in $1.5 \%$ agar gels containing GelGreen stain (Biotium, Hayward, CA). Positive and negative controls were included in each PCR assay. PCR products with appropriate sizes of amplicons were purified using a QIAquick PCR Purification Kit (Qiagen, Valencia, CA) according to manufacturer's instructions, then sequenced in both directions using ABI 3130 Genetic Analyzer (Applied Biosystems, Foster City, CA).

\section{Genome analysis}

Whole genome sequencing (WGS) was performed using the Illumina MiSeq instrument. Sequencing of the two samples resulted in 8 million 51 nucleotide long sequencing reads. The sequencing reads were filtered to exclude: (a) nucleotides below the quality threshold of 0.05 (using modified Richard Mott algorithm); (b) unknown nucleotides; and (c) sequencing library adapters. The reads were then assembled allowing for up to $5 \%$ mismatches using CLC Genomics Workbench De Novo Assembly tool (http://www.clcbio.com) and the original sequenced reads were mapped back to the assembled contigs (Table 1). Rapid Annotation using Subsystem Technology tool kit (RASTtk) was used to cut open reading frames from the assembled contigs on the PATRIC web interface. ${ }^{9}$ Annotated features were clustered together in protein space using CD-HIT with the following parameters: $70 \%$ global identity and $70 \%$ alignment coverage. ${ }^{10}$ Figure 1 shows a Venn diagram of the feature clustering between the two sequenced isolates and the closest reference genome (Janibacter terrae NBRC $107854=$ JCM 12887). Blastn was used to compare the features annotated with the closest reference, Janibacter terrae NBRC $107854=$ JCM 12887 (GCA_001598955.1). ${ }^{11}$ The results were visualized using Blast Ring Image Generator (Figure 2). ${ }^{12}$

\section{Results}

A 60-year-old previously healthy female experienced symptoms of headache, dizziness and syncope episodes, general weakness, exertional fatigue, and shortness of breath for 2 years. She also reported lowgrade episodic fevers in the preceding 6 months. One month prior to hospital admis- sion, the patient's fevers became more persistent and she was evaluated in an outpatient clinic. She was prescribed empiric antibiotics of unknown type, but did not improve. She was admitted to the intensive care unit in May 2014 due to periodic squeezing pain in the chest that radiated under the left shoulder blade with the original diagnosis of endocarditis. The patient lived in a rural area near the town of Kareli in the region of Shida Kartli, Georgia (South Caucasus). She was engaged in agricultural work and reported frequent contacts with farm animals (pigs and cattle) and pets at home.

Clinical signs upon hospital admission included fever of $38^{\circ} \mathrm{C}$, ischemic disease, angina, and arterial hypertension. No rash or lymphadenopathy was reported. Coronary angiographyshowed unaltered coronary vessels. Echocardiography detected critical stenosis of the aortic valve with high gradient and critical opening. On auscultation there was rough systolic murmur at every auditory point, with epicenter on the aorta. Heart rate -76 beats/min, pulse arrhythmic, average filling and tension. T/A $-130 / 60 \mathrm{mmHg}$. The patient's WBC was $5.6010^{3} / \mathrm{uL}$ (normal range: $3.4-9.6$ $10^{3} / \mathrm{uL}$ ), neutrophil count $42 \%$ (normal range: $40 \%-60 \%$ ), and lymphocyte count $48 \%$ (normal range: $20 \%-40 \%$ ), platelets - $19310^{3} / \mathrm{uL}$ (normal range: $157-371$ $10^{3} / \mathrm{uLfor}$ females); and ESR - $10 \mathrm{~mm} / \mathrm{h}$ (normal range: $1-20 \mathrm{~mm} / \mathrm{h}$ for females). Chest X-ray and abdominal ultrasound did not show any abnormalities. The case was diagnosed as aortic stenosis, and the patient underwent heart valve replacement. As standard post-surgical prophylaxis, the patient was treated with Ceftriaxone 1 gram two times per day after the surgery.

No bacterial growth was observed on agar plates inoculated with blood of the patient. On the seventh day after inoculation of agar plates with homogenized cardiac tissues two small bacterial colonies were observed. Two slow-growing colonies with slightly different morphology were observed on the agar plate inoculated with heart tissues. Both colonies had circular shape and light cream color with a slight difference in size. No hemolysis on chocolate agar was observed. These two colonies were separately sub-cultured onto new agar plates. Microscopy indicated Gram-positive coccobacilli. When bacterial mass on both plates was sufficient, colonies were harvested on brain heart infusion medium and stored at $-70^{\circ} \mathrm{C}$ until the strains were shipped to CDC-Fort Collins for additional characterization.

By Mega BLAST search, the ribosomal amplicons from both isolates were identified as a Janibacter sp. based on the fragment amplification and sequence analysis with the highest match to Janibacter hoylei. The 16S rRNA sequences from both isolates were undistinguished.

Sequencing of each isolate genome resulted in 2 and 6 million sequencing reads 51 nucleotides long in size trimmed to 32 bases long non-overlapping subsequences with a minimum threshold quality of 10 . To determine the presence and identity of microbial contaminants, sequencing data was tested against a collection of 13,774 complete bacterial, viral, plasmid, phage, and fungal reference genome sequences, which included all reference genomes available from the NCBI Reference Sequence (collection as of November 2013).

Intrasporangiaceae family had the highest number of identified genes from the database. A more detailed mapping to every genome entry in NCBI under the Intrasporangiaceae family was performed. For references, which did not contain a fully assembled genome, the contigs were concatenated into one long sequence that was treated as a genome. Lowest Common Ancestry analysis of full length $16 \mathrm{~S}$ sequences assembled from whole genome sequencing reads showed a $99.9 \%$ match to the genus Janibacter. ${ }^{13}$ The closest match by genome analysis was to Janibacter ter-

Table 1. Assembly statistics genomes of two strains of Janibacter (TB1 and TB2)

\begin{tabular}{lcc} 
& Strain TB1 & Strain TB2 \\
N75 & 20,309 & 22,383 \\
N50 & 35,574 & 48,911 \\
\hline N25 & 56,767 & 84,095 \\
Minimum Contig Length & 205 & 210 \\
\hline Maximum Contig Length & 118,653 & 165,607 \\
Average Contig Length & 15,154 & 22,752 \\
\hline Number of Contigs & 219 & 142 \\
Total Bases Assembled & $3,318,728$ & $3,230,833$ \\
\hline
\end{tabular}


rae. ${ }^{14}$ The similarity between the both strains and $J$. terrae was calculated as a Jaccard distance measured by proportion between a number of shared genes by average number of genes between two compared genomes. According to the calculation, the strain TB1 was $84.4 \%$ similar to $J$. terrae, while the strain TB2 was $87.9 \%$ similar to J. terrae. Comparison of the genomes of TB1 and TB2 has revealed some polymorphic differences. The gene clustering results confirmed an approximately $15 \%$ difference in the gene composition between the two stains (Figure 1).

\section{Discussion}

Two discoveries can be highlighted from the reported results: 1) both bacterial cultures isolated from a human patient with aortic stenosis were identified as a species of genus Janibacter that has never been previously reported as a source of a cardiac disease in humans or animals; and 2) complete genome comparison indicated genomic difference between bacterial cultures obtained from the same cardiac tissues.

The report of the isolation of two closely related bacterial strains belonging to the genus of Janibacter from the case of aortic stenosis is novel not only for this region, but presents the first known case worldwide. The bacteria of genus Janibacter were originally found in the environment,${ }^{15}$ but since
2005 at least nine cases of human infections associated with these microorganisms have been reported. Among these cases, one case was associated with $J$. melonis, ${ }^{16}$ one case with $J$. hoylei, ${ }^{17}$ two cases with an undescribed species of Janibacter, ${ }^{18,19}$ and five cases with $J$. terrae. ${ }^{20,21}$ The cases associated with $J$. terrae are especially interesting since the isolates that we report here from the cardiac tissues were closely related by the genomic analysis to this species of Janibacter. Fernandez- Natal et al..$^{20}$ reported cases of infection by $J$. terrae in four febrile patients with severe underlying conditions in Spain. All four patients were treated with antibiotics, two of them with favorable outcome, and two severely immunocompromised patients died. More recently, Wan et $a l^{21}$ reported a case of bilateral psoas abscess caused by J. terrae.

Although this is the first report of bacteria of the genus Janibacter isolated from a cardiac valve, these microorganisms were broadly described as "coryneform" bacteria with some representatives being reported as sources of endocarditis. ${ }^{22}$ Coryneform bacteria, defined as non-sporing Gram-positive rods with an irregular outline, were historically dismissed as a part of the normal human skin flora when recovered from human patients, but have increasingly been implemented as causes of significant infections in clinical practice..$^{23}$

Identification of a Janibacter strain in cardiac tissue can lead to evaluation of this bacteria as a potential source of infection; however, we wish to keep a conservative approach for a validation of this claim. Cardiac valve tissue is considered sterile; nevertheless, though a contamination of cardiac tissue during the surgery or culturing procedure is very unlikely, we cannot rule out such a possibility. More importantly, we cannot prove a causal connection between this bacterium and pathogenic manifestations in the patient's heart because of very limited information available about these bacteria. Therefore, an etiological role of this bacterium as a source of aortic stenosis requires further investigations. Nevertheless, the presented findings suggest that clinicians should be aware of a possible risk of infection by bacteria of the genus Janibacter with the ability to cause invasive cardiac disease.

Having limited data on potential risk factors, it is hard to speculate about routes of transmission of the bacteria to the patient. The patient's report of frequent exposure to farm animals, however, may suggest a possible zoonotic source of the infection. In Germany, Janibacter DNA was identified from a biofilter treating air emissions from a livestock facility. ${ }^{24}$

The study has demonstrated additional perspectives based on the ability of the computational analysis of the whole bacterial genomes to identify and characterize fastidious microorganisms discovered in clinical specimens. Although 16SrRNA analysis

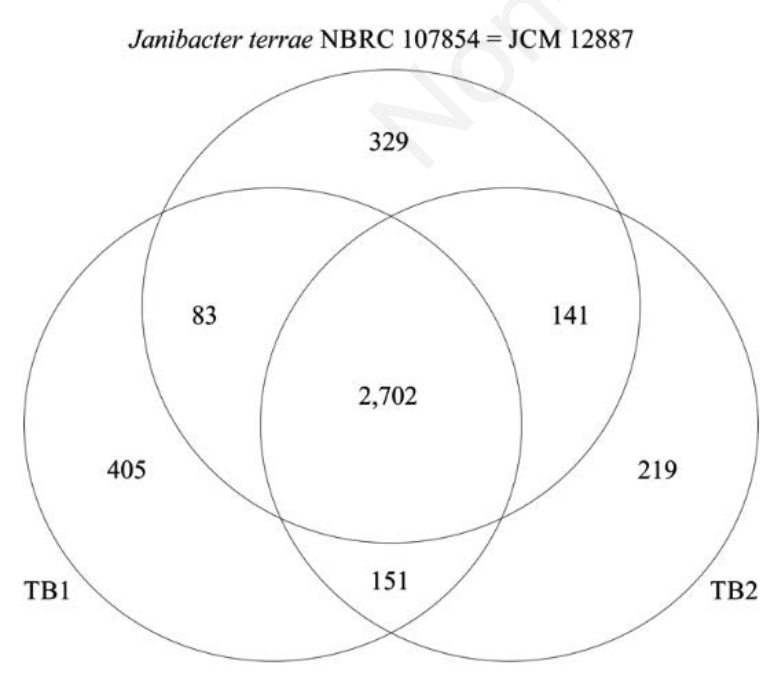

Figure 1. Venn diagram comparison of gene composition between strains TB1 and TB2 cultured from valve tissues of a patient with aortic stenosis, and Janibacter terrae.

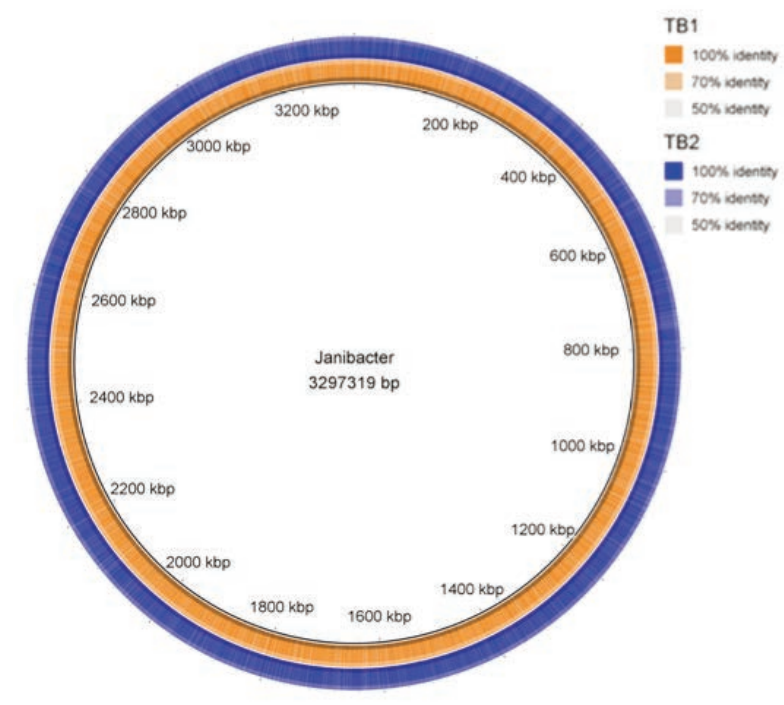

Figure 2. Ring image displaying the similarity of the two isolates from valve tissues of a patient with aortic stenosis with Janibacter terrae (strain NBRC $107854=$ JCM 12887). 
identified Janibacter species in the culture colonies, the whole genome sequencing allowed to more precisely differentiate these strains from other Janibacter species and to evaluate differences between colonies. The latter finding highlighted the fact that the bacterial population occupying cardiac tissues from one patient was polymorphic. Genomic comparison of cultures of Janibacter obtained from human cases and from environmental sources presents a promising direction for evaluating a role of these bacteria as human pathogens.

\section{References}

1. Brouqui P, Raoult D. Endocarditis due to rare and fastidious bacteria. Clin Microbiol Rev 2001;14:177-207.

2. Subedia S, Jenningsa Z, Chen SC. Laboratory approach to the diagnosis of culture-negative infective endocarditis. Heart Lung Circulation 2017;8:763-71.

3. Gersony WM, Hayes CJ, Driscoll DJ, et al. Bacterial endocarditis in patients with aortic stenosis, pulmonary stenosis, or ventricular septal defect. Circulation 1993;87:1121-6.

4. Cohen DJ, Malave D, Ghidoni JJ, et al. Role of oral bacterial flora in calcific aortic stenosis: an animal model. Ann Thorac Surg 2004;77:537-43.

5. Higuchi ML, Higuchi-Dos-Santos MH, Pierri H, et al. Mycoplasma pneumoniae and Chlamydia pneumoniae in calcified nodules of aortic stenotic valves. Rev Inst Med Trop Sao Paulo 2002;44: 209-12.

6. Pierri H, Higuchi-dos-Santos MH, Higuchi Mde L, et al. Density of Chlamydia pneumoniae is increased in fibrotic and calcified areas of degenerative aortic stenosis. Int J Cardiol 2006; 108:43-7.

7. Schnitzer K, Or Z, Sawaed S, et al. Rapidly Progressive Bioprosthetic aortic valve stenosis due to Bartonella species endocarditis. Ann Thorac Surg 2017;104:57-9.

8. Kandelaki G, Malania L, Bai Y, et al. Human lymphadenopathy caused by ratborne Bartonella, Tbilisi, Georgia. Emerg Inf Dis 2016;22:544-6.

9. Wattam AR, Abraham D, Dalay O, et al. PATRIC, the bacterial bioinformatics database and analysis resource. Nucl Acids Res 2014;42:581-91.

10. Fu L, Niu B, Zhu Z, et al. CD-HIT: accelerated for clustering the next-generation sequencing data. Bioinformatics 2012;28:3150-2.

11. Camacho C, Coulouris G, Avagyan V, et al. BLAST+: architecture and applications. BMC Bioinformatics 2018;10: 421.

12. Alikhan NF, Petty NK, Ben Zakour NL, et al. BLAST Ring Image Generator (BRIG): simple prokaryote genome comparisons. BMC Genomics 2011;12: 402.

13. Pruesse E, Peplies J, Glöckner FO. SINA: accurate high-throughput multiple sequence alignment of ribosomal RNA genes. Bioinformatics 2012;28: 1823-9.

14. Yoon JH, Lee KC, Kang SS, et al. Janibacter terrae sp. nov., a bacterium isolated from soil around a wastewater treatment plant. Int J Syst Evol Microbiol 2000;50:1821-7.

15. Martin K, Schumann P, Rainey FA, et al. Janibacter limosus gen. nov., sp. nov., a new actinomycete with meso- diaminopimelic acid in the cell wall. Int J Syst Bacteriol 1997;47:529-34.

16. Elsayed S, Zhang K. Bacteremia caused by Janibacter melonis. J Clin Microbiol 2005;43:3537-9.

17. Lim YK, Kweon OJ, Kim HR, et al. First case of bacteremia caused by Janibacter hoylei. APMIS 2017;125: 665-8.

18. Loubinoux J, Rio B, Mihaila L, et al. Bacteremia caused by an undescribed species of Janibacter. J Clin Microbiol 2005;43:3564-6.

19. Worodria W, Anderson J, Cattamanchi A, et al. The role of speciation in positive Lowenstein-Jensen culture isolates from a high tuberculosis burden country. PLoS One 2011;6:e27017.

20. Fernández-Natal MI, Sáez-Nieto JA, Medina-Pascual MJ, et al. First report of bacteremia by Janibacter terrae in humans. Infection 2015;43:103-6.

21. Wan WY, Mughal A, Ward K. Bilateral psoas abscess caused by Janibacter terrae, an unusual condition and organism resulting in laboratory and management conundrums. Acta Clin Belg 2016;19:14.

22. Graevenitz A. Importance of Coryneform Bacteria in Infective Endocarditis. Infect Dis Rep 2015;7: 6103.

23. Bernard K. 2012. The genus Corynebacterium and other medically relevant coryneform-like bacteria. J Clin Microbiol 2012;50:3152-8.

24. Kristiansen A, Lindholst S, Feilberg A, et al. Butyric acid- and dimethyl disulfide-assimilating microorganisms in a biofilter treating air emissions from a livestock facility. Appl Environ Microbiol 2011;77:8595-604. 